\title{
The role of vascular mimicry as a biomarker in malignant melanoma: a systematic review and meta-analysis
}

Zhenhua Zhang ${ }^{1 \dagger}$, Saber Imani ${ }^{1+}$, Marzieh Dehghan Shasaltaneh ${ }^{2}$, Hossein Hosseinifard ${ }^{3}$, Linglin Zou', Yu Fan ${ }^{1}$ and Qinglian Wen ${ }^{1 *}$

\begin{abstract}
Background: Vasculogenic mimicry (VM) a microvascular system consisting of non-endothelial cells that is newly formed by aggressive tumors, has been proposed as an important therapeutic target in malignant melanoma (MM). We performed a systematic literature review to evaluate the diagnostic and prognostic accuracy of VM status for overall survival of MM patients.

Methods: The quality of the included studies was evaluated using the QUADAS-2 tool. Diagnostic capacity of VM variables, including sensitivity, specificity, positive likelihood ratio (PLR), negative likelihood ratio (NLR), diagnostic odds ratio (DOR), and the area under summary receiver operating characteristic (SROC), were pooled using MetaDiSc software.

Results: A retrospective observational study was conducted based on twelve clinical studies including 978 clinically confirmed melanoma patients with proportion (P). VM+ melanoma cells were associated with poor prognosis in $38 \%$ of MM group ( $P=0.35,95 \%$ confidence intervals $(\mathrm{Cl})$ : $0.27-0.42, p<0.001)$. The pooled sensitivity and specificity were 0.82 (95\% Cl: $0.79-0.84)$ and 0.69 (95\% Cl: 0.66-0.71), respectively. Furthermore, the pooled PLR, NLR, and DOR were 2.56 (95\% Cl: 1.94-3.93), 0.17 (95\% Cl: 0.07-0.42), and 17.75 (95\% Cl: 5.30-59.44), respectively. Furthermore, the AUC of SROC was 0.63 , indicating high reliability of VM status as a biomarker. Importantly, subgroup results suggested that VM+ status is a significantly accurate prognostic biomarker when diagnosed by the CD31-/PAS+ staining methods in Asian MM samples ( $p<0.001)$.

Conclusions: Our findings support the potential of VM status of tumors as a promising prognostic biomarker and emphasize an effective adjuvant therapeutic strategy in the prognosis of Asian MM patients.
\end{abstract}

Keywords: Vasculogenic mimicry, Malignant melanoma, Diagnosis, Prognosis, Meta-analysis

\section{Background}

Malignant melanoma (MM) is the most aggressive skin cancer and the most common skin disorder in Caucasians characterized by aggressive and progressive disease states, leading to major cancer-related morbidity and mortality, with an estimated global incidence of about 200,000 new cases per year and 50,000 cancer-related deaths in 2018 [1-3]. The incidence of MM has been rapidly increasing

\footnotetext{
* Correspondence: wq173115@hotmail.com

${ }^{\dagger}$ Zhenhua Zhang and Saber Imani contributed equally to this work.

'Department of Oncology, The Affiliated Hospital of Southwest Medical

University, Luzhou City, Sichuan Province 646000, People's Republic of China

Full list of author information is available at the end of the article
}

over the last 10 years in the Asian and Mediterranean population and in Singapore is diagnosed as the seventh and the eighth most common cancer among men and women, respectively [3-6]. Nevertheless, the Asian population has a notably lower risk of MM than Caucasians due to ethnic differences, anatomic distribution, histologic subtypes, and stage at diagnosis [6, 7]. Interestingly, Ultraviolet (UV) radiation, race, lifestyle, and genetic differences are the most important reasons for the high mortality rate of melanoma which can be decreased via early-stage detection and prevention [8-13]. Dermoscopy and intrinsic molecular subtyping of melanoma have been widely accepted as accurate diagnostic methods with more

(c) The Author(s). 2019 Open Access This article is distributed under the terms of the Creative Commons Attribution 4.0 International License (http://creativecommons.org/licenses/by/4.0/), which permits unrestricted use, distribution, and 
than $50 \%$ accuracy compared with the clinical diagnosis in patients with MM [14, 15].

Recent investigations identified a new non-angiogenesisdependent pathway entitled vasculogenic mimicry (VM), which refers to a vessel-like structure formed by extremely aggressive tumor cells that imitate endothelial cells [16, 17]. VM has been considered as a cancer hallmark that can independently facilitate tumor neovascularization by the formation of fluid-conducting and vascular endothelial cells [18-20]. VM could dedifferentiate into numerous cellular phenotypes and obtain endothelial-like features, resulting in the formation of the de novo matrix-rich vascular-like network, such as plasma and red blood cells [21, 22]. The co-generation of endothelial cells, channels, laminar structures, and heparin sulfate proteoglycans are the main pathophysiological characteristics of VM in human melanoma patients [23-25]. Aggressive VM+ tumor cells are characterized by a higher expression of the basement membrane extracellular matrix (ECM) components laminin5 2 and metalloproteinases (MMPs) $-1,-2,-9$, and -14 $[21,22,26]$. In highly aggressive melanoma cells downregulation of vascular endothelial cadherin and upregulation of ECM components promotes the perfusion of the VM pathway $[19,21]$. Ultimately, the VM+ melanoma cells are associated with more aggressive and metastatic tumor biology.

Accumulating evidence suggests that VM is associated with poor prognosis in various malignant human tumors, including breast [27], colorectal [28], prostate [29], hepatocellular carcinoma [30], lung [29], ovarian [31], gastric [32], and bladder cancers [33]. Despite numerous experimental studies, the prognostic value of VM status for survival in MM patients is still controversial and inconclusive. Understanding the role of VM in MM pathogenesis may help to develop effective treatments for tumor invasion and to overcome drug resistance in MM [34].

Hence, we conducted a quantitative systematic review along with a comprehensive meta-analysis investigation based on eligible studies to resolve inconsistent and often ambiguous findings. Furthermore, we identified the prognostic accuracy of VM status in cancer patients to predict other clinical pathological feature outcomes of MM.

\section{Methods}

This systematic review and meta-analysis was performed according to the recommendations of the Preferred Reporting Items for Systematic Reviews and Meta-Analyses (PRISMA) statement guidelines [35].

\section{Search strategy and study selection}

MEDLINE electronic databases of Pubmed, Embase, Wiley Online Library, Web of Science, Science Direct, Cochrane library, and VIP-Google Scholar were searched to assess the prognostic value of $\mathrm{VM}$ in melanoma patients prior to
April 18, 2019. Different spelling and synonyms were combined applying Boolean "OR" and main terms were linked applying Boolean "AND" to identify all relevant studies. The search string was conducted using $\mathrm{MeSH}$ terms and following main headline terms or free words based on the research question (both the UK and US spellings), such as: "vascular mimicry OR vasculogenic mimicry OR tumor cell-lined vessels OR tumor-derived endothelial cells" AND "prognosis OR survival OR outcome" AND "melanoma OR basal cell carcinoma OR squamous cell carcinoma OR cancer OR neoplasms OR malignant melanoma OR basal-cell skin OR squamous-cell skin OR skin". The comprehensive literature search strategies are detailed in Additional file 1: Table S1, which were separately retrieved and screened by four researchers (ZZ, SI, HH, and MDS).

\section{Inclusion/exclusion criteria}

The current meta-analysis covered all prospective and randomized controlled trials (RCTs) that were considered eligible if they met the following criteria: $(i)$ Melanoma patients were confirmed by immunohistochemical or histochemical tests. (ii). $\mathrm{VM}+$ tumor tissue samples were assessed by classical staining of the specimens, including positive Periodic Acid-Schiff (PAS) and/or negative endothelial cell markers, CD34 or CD31; (iii) No previous systemic treatment for metastatic disease. Likewise, we excluded all non-comparative, review, case-control, conference abstracts, meeting reports, commentaries, and unrelated articles, as well as family-based, in vitro, and animal studies. Moreover, we excluded duplicate studies, continued work of previous publications, and poor quality studies, as well as those with incomplete and/or missing data such as sample size and VM frequency.

\section{Data extraction and quality assessment}

All selected articles were reviewed independently by three researchers (ZZ, SI, and MDS) according to the population, intervention, control, and outcomes (PICO) principle [36] and any disagreements or inconsistencies in a search process were addressed through consultations and debate. If an acceptable consensus was not reached, a fourth partner (QW) would resolve these disagreements based on the original data. The key demographics and clinicopathological information of all qualified data collections were summarized in Tables 1 and 2. These included the first author's name, publication year, total cases, gender, country of origin population, age, follow up time, VM+ or VM- rate, analyzing methods of VM, Clark level, and location of sampling. In addition, we emailed corresponding authors to obtain any additional or missing information, as well as original data needed for the meta-analysis. If the above data were not cited in the original study or no reply was received by email, the item was reported as "not reported (NR)". 
Table 1 Demographic information of included studies

\begin{tabular}{|c|c|c|c|c|c|c|c|c|}
\hline \multirow{2}{*}{$\begin{array}{l}\text { First author } \\
\text { (Ref.) }\end{array}$} & \multirow[t]{2}{*}{ Year } & \multirow[t]{2}{*}{ SS } & \multirow{2}{*}{$\begin{array}{l}\text { Gender } \\
(\mathrm{M} / \mathrm{F})\end{array}$} & \multirow{2}{*}{$\begin{array}{l}\text { Population } \\
\text { (ethnicity) }\end{array}$} & \multicolumn{2}{|l|}{ Age } & \multirow{2}{*}{$\begin{array}{l}\text { Flow } \\
\text { time } \\
\text { (months) }\end{array}$} & \multirow[t]{2}{*}{ NOS } \\
\hline & & & & & $\leq 30$ & $>30$ & & \\
\hline Maniotis AJ, [34] & 1999 & 234 & NA & USA (C) & NA & NA & 480 & 7 \\
\hline Massi D, [35] & 2004 & 45 & $22 / 23$ & Italy (C) & - & 45 & 120 & 8 \\
\hline Hillen F, [37] & 2008 & 58 & $18 / 40$ & Netherland (C) & 16 & 42 & 120 & 8 \\
\hline Zhang SH, [38] & 2009 & 124 & $67 / 57$ & China (A) & NA & NA & 250 & 6 \\
\hline Shi L, [39] & 2010 & 45 & $31 / 14$ & China (A) & - & 45 & 100 & 7 \\
\hline Beurden BV, [40] & 2012 & 123 & $58 / 65$ & Netherland (C) & 41 & 82 & 200 & 9 \\
\hline Itzhaki O, [41] & 2013 & 15 & $10 / 5$ & Israel (C) & 1 & 14 & NA & 7 \\
\hline Song H, [42] & 2015 & 62 & $34 / 28$ & China (A) & NA & NA & 90 & 7 \\
\hline Baocun S, [43] & 2015 & 60 & NA & China (A) & NA & NA & 39 & 8 \\
\hline Zhao X, [44] & 2015 & 79 & $47 / 32$ & China (A) & NA & NA & 200 & 7 \\
\hline Liang $X,[45]$ & 2017 & 81 & $54 / 27$ & China (A) & 59 & 22 & 100 & 7 \\
\hline Zhang W, [46] & 2017 & 52 & $36 / 16$ & China (A) & 37 & 15 & 80 & 7 \\
\hline
\end{tabular}

Abbreviations: Ref. Reference, SS Sample size, M Male, F Female, C Caucasian, A Asian, NOS Newcastle-Ottawa scale, NA Not avalibale

${ }^{a}$ The quality of non-randomized studies will be appraised using the Newcastle-Ottawa scale (NOS), categorized into three groups: the selection of the study

groups; the comparability of the groups; as well as the ascertainment of either the exposure or outcome of interest for case-control or cohort studies respectively

All eligible studies were assessed based on the NewcastleOttawa scale (NOS) [37] and Quality Assessment of Diagnostic Accuracy Studies 2 (QUADAS-2) [38] protocols. In addition, the probability of bias was calculated based on the criteria from the Cochrane Collaboration's tool (Cochrane handbook for systematic reviews of interventions version 5.1.0.).

\section{Statistical analysis}

The current systematic meta-analysis was carried out applying Comprehensive Meta-Analysis (CMA) software (Biostat, Englewood, NJ 07631, USA, version 2.2.064). The diagnostic accuracy and ROC curves were conducted on
MetaDiSc (version 1.4). Additionally, the quality of study was calculated by RevMan version 5.2 [39, 40]. Pooled specificity, pooled sensitivity, negative likelihood ratio (NLR), positive likelihood ratio (PLR), and diagnostic odds ratio (DOR) were calculated with corresponding $95 \% \mathrm{CIs}$ to evaluate the diagnostic value of VM. Furthermore, the summary receiver operating characteristic (SROC) curve was calculated for the involved studies with an overall area under the curve (AUC). Results of the meta-analysis were reported as a proportion (P) with $95 \%$ confidence intervals (CIs). All data were reported as mean \pm standard deviation (SD) or as median (range). As well, a description of qualitative variables as number and percentage are given. The

Table 2 Main clinicopathological and vasculogenic mimicry characteristics of all relevant studies

\begin{tabular}{|c|c|c|c|c|c|c|c|c|c|}
\hline \multirow{2}{*}{$\begin{array}{l}\text { First author } \\
\text { (Ref.) }\end{array}$} & \multicolumn{2}{|c|}{ VM state $\mathrm{n}(\%)$} & \multirow{2}{*}{$\begin{array}{l}\text { Methods } \\
\text { of VM } \\
\text { assay }\end{array}$} & \multicolumn{3}{|c|}{ Clark level $^{a}$} & \multicolumn{3}{|l|}{ Location } \\
\hline & $\mathrm{VM}+$ & VM- & & III & IV & V & Head and neck & Trunk & Extremities \\
\hline Maniotis AJ, [34] & $106(45)$ & $128(55)$ & PAS & - & 234 & - & 234 & - & - \\
\hline Massi D, [35] & $15(30)$ & $30(70)$ & CD31/PAS & - & 45 & - & 6 & 21 & 18 \\
\hline Hillen F, [37] & $22(38)$ & $36(78)$ & PAS & 12 & 22 & 24 & 6 & 17 & 22 \\
\hline Zhang SH, [38] & $54(43)$ & $70(57)$ & PAS & - & 65 & 59 & 16 & 46 & 62 \\
\hline Shi L, [39] & $11(27)$ & $34(73)$ & CD31/PAS & 33 & 12 & - & 32 & 13 & - \\
\hline Beurden BV, [40] & $42(34)$ & $81(66)$ & PAS & 23 & 51 & 49 & - & 112 & 11 \\
\hline Itzhaki O, [41] & $14(93)$ & $1(7)$ & CD31/PAS & 2 & 8 & 5 & 2 & 10 & 3 \\
\hline Song H, [42] & $25(41)$ & $37(59)$ & CD31/PAS & 14 & 45 & 3 & 62 & - & - \\
\hline Baocun S, [43] & $10(5)$ & $50(95)$ & CD31/PAS & - & 38 & 22 & NA & NA & NA \\
\hline Zhao X, [44] & $36(45)$ & $43(55)$ & CD34/PAS & 46 & 33 & - & 21 & 43 & 15 \\
\hline Liang X, [45] & $35(43)$ & $46(57)$ & CD34/PAS & - & 41 & 40 & 34 & 28 & 19 \\
\hline Zhang W, [46] & $106(45)$ & $128(55)$ & CD34/PAS & - & 22 & 30 & 22 & 16 & 14 \\
\hline
\end{tabular}

Abbreviations: Ref. Reference, VM Vasculogenic mimicry, PAS Periodic acid schiff's, NA Not available

${ }^{a}$ All tissue samples are formalin-fixed, paraffin-embedded and categorized by five anatomical levels of Clark's staging system 
chi square-based Q-test was applied to testify betweenstudy heterogeneity. Subgroup analysis was performed to identify the source of existing heterogeneity between the $\mathrm{VM}+$ and available sub analyses such as sample size, race, and VM detection methods. Publication bias was assessed using Begg's funnel plots [43] and Egger's regression test [44]. A value of " $P r>|z|$ " less than 0.05 was considered to be a potential publication bias. All reported $p$ values were two-sided and $p<0.05$ was considered statistically significant.

\section{Results}

\section{Description of studies}

A detailed PRISMA flowchart of the study identification, screening, and exclusion process is shown in Fig. 1. The primary manual search yielded 426 potentially eligible publications through searching of electronic databases and 1 record. After excluding duplicate studies (198 studies), 229 publications were kept for screening, of which 102 records were excluded according to the inclusion and exclusion criteria for database searching. Then, the remaining 127 articles were further assessed by abstract reviewing, and 67 studies were discarded being either cell or animal studies. Following careful review of titles and abstracts, full-text articles of 60 studies were assessed for suitability. Twenty studies were excluded for obvious irrelevance, 16 studies were precluded for dealing with other types of cancer, and 12 studies were dismissed due to having no related assays. Finally, 12 studies were retained in this meta-analysis [42, 45-54].

\section{Characteristics of studies}

The demographic information of all relevant studies is detailed in Table 1 . According to this table, a total of 12 studies with $978 \mathrm{MM}$ patients dating between 1999 and 2017 were included in this systematic review and metaanalysis. Seven studies were conducted in people of the Asian race (58.4\%) [41, 42, 49, 50, 52-54], four studies (33.4\%) in European countries [45, 48, 51, 55], one study in the USA (8.2\%) [47], and no study from African populations. Gender subgroups among the 978 patients included 377 male and 307 female patients. The major clinicopathological features of the included studies are shown in Table 2. More than $80 \%$ of the MM patients were diagnosed by histopathological tests. PAS staining combined with endothelial markers (CD31 or CD34) is a commonly used method for identification of tumor VM in paraffin-embedded tissue specimens and was done in 8 studies (66.7\%) [41, 42, 45, 50, 52-55], as well as PAS staining in 4 studies $[47-49,51]$. Moreover, significant

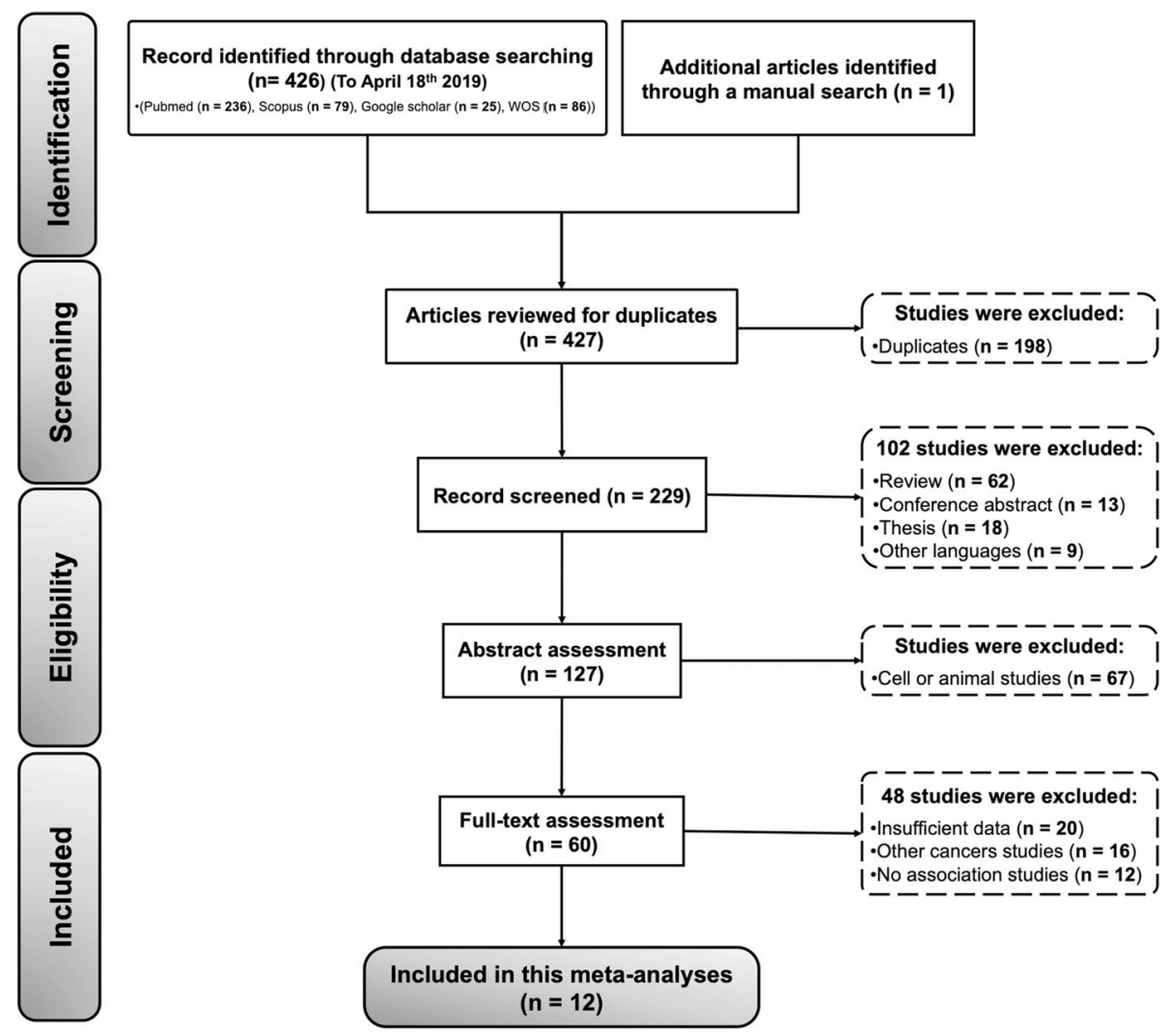

Fig. 1 Flow diagram of included studies (following PRISMA guidelines, $n=$ number of studies) 
predictors of $\mathrm{VM}+$ in both adjusted and unadjusted analyses were Clark level IV or V (84.4\%). Finally, 11 studies reported the association between VM and clinicopathological parameters regarding OS [41, 42, 45-54], with the follow-up period ranging from 39 to 480 months.

\section{Quality assessment}

All 12 papers were methodologically assessed according to NOS and QUADAS-2 quality evaluation standards of the Cochrane Reviewer handbook. Both systems' tools focused on the study dependent on the methodology. Overall, the average NOS score was approximately 7.4 out of 12, which could be classified nearly in the high quality group. For each study, the NOS score is given in Table 1. Furthermore, QUADAS-2 results confirmed that no significant bias was detected in the present meta-analysis. Details of the quality evaluation of eligible studies according to the NOS score are summarized in the Additional file 1: Table S3. The reviewers' decisions about each risk of bias and applicability concerns graph are presented as percentages across selected studies. Additional file 2: Figure S1 shows all parameters of QUADAS-2 assessment individually. In this study, no significant bias and applicability concerns were found in any of the selected studies.

\section{Outcome of the meta-analysis}

The relationship between $\mathrm{VM}+$ and overall survival of MM patients was identified applying the pooled proportions test method. We used a random effect approach because the heterogeneity of the overall prognosis was relatively high, which is shown across the study $\left(\mathrm{I}_{2}=\right.$ 79.8, p-value $<0.001)$. Based on heterogeneous cross of 12 studies, VM was associated with poor prognosis in $38 \%$ of MM group compared to the VM-group $(P=0.35$,
95\% confidence intervals (95\% CIs): $0.27-0.42$, p-value $<$ 0.001 ). Therefore, these results suggested that $\mathrm{VM}+$ indicated a poorer prognosis for MM patients (Fig. 2).

\section{Diagnostic accuracy}

The effect of heterogeneity on the diagnostic threshold was evaluated based on the Spearman correlation coefficient. Figure 3 presents the forest plots of pooled sensitivity and specificity, with the $95 \%$ CIs for individual studies. According to the results, the overall pooled sensitivity of $\mathrm{VM}+$ tumor was 0.82 (95\% CI: 0.79-0.84, Fig. 4a), while the specificity of VM+ tumor was 0.69 (95\% CI: 0.66-0.71; Fig. 4b), among the 12 included studies. Furthermore, the overall pooled results for PLR, NLR, and DOR were 2.56 (95\% CI: 1.94-3.93), 0.17 (95\% CI: 0.07-0.42), and 17.75 (95\% CI: 5.30-59.44), respectively.

\section{Subgroup analysis}

Associations between $\mathrm{VM}+$ and the possible demographic and clinicopathological features of MM patients are listed in Table 3. According to the results, none of the above covariates contributed to the heterogeneity (all $p>0.05$ ). Therefore, according to those covariates, the pooled sensitivity, specificity, PLR, NLR, DOR, and AUC were measured for significant sub-analysis parameters. We detected statistically significant relationships between VM and sample size, VM and race, as well as between VM expression and staining method (Fig. 5). As shown in Fig. 5a and Table 3, VM+ is a potentially accurate prognostic biomarker in CD31-/PAS+ $(P=0.24$, 95\% CI: 0.15-0.35) compared to CD34-/PAS+ $(P=0.39$, 95\% CI: $0.27-0.42)$ and the PAS+ staining subgroups $(P=0.40,95 \%$ CI: $0.30-0.52)$. As a result, the CD31 -/PAS+ staining method is a relatively accurate diagnostic method for detection of the VM, with $75 \%$ sensitivity

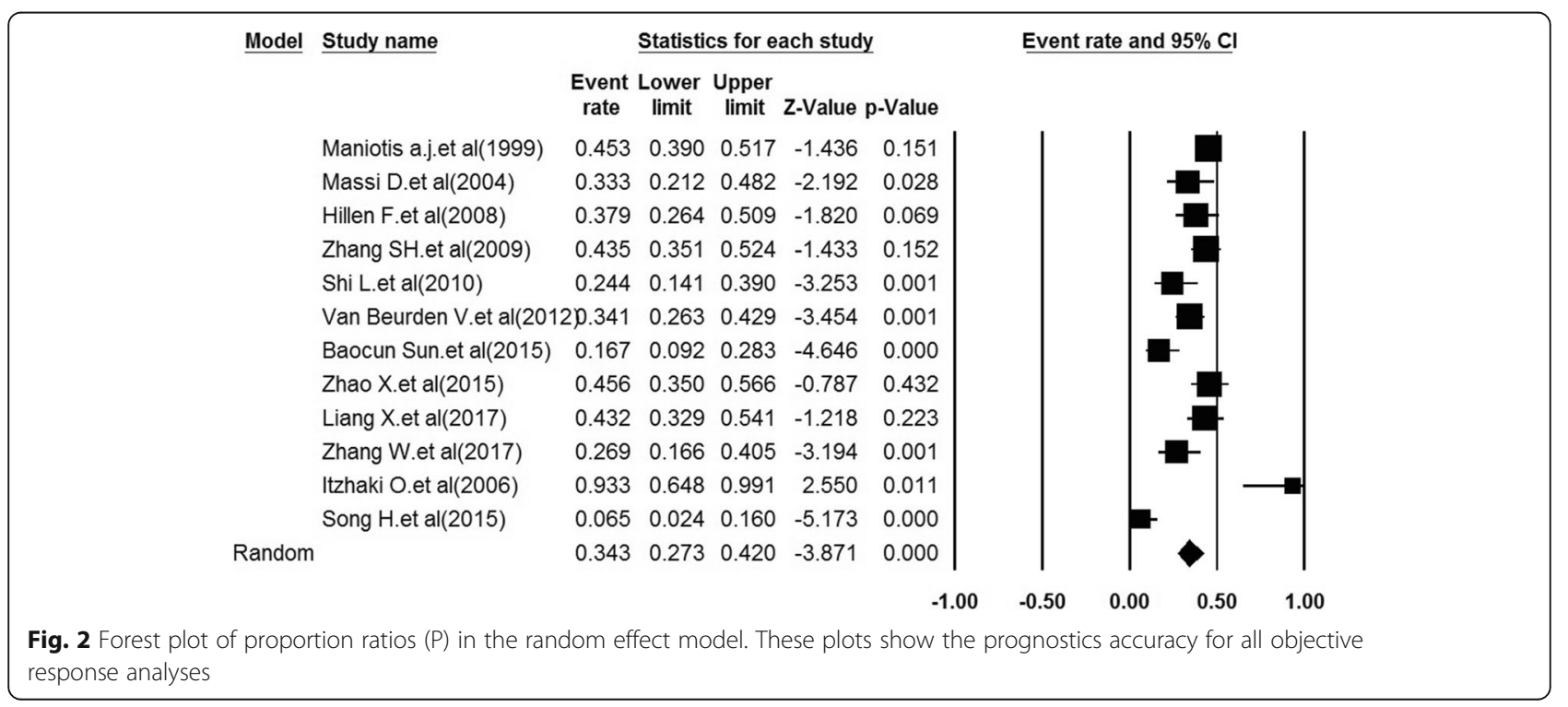




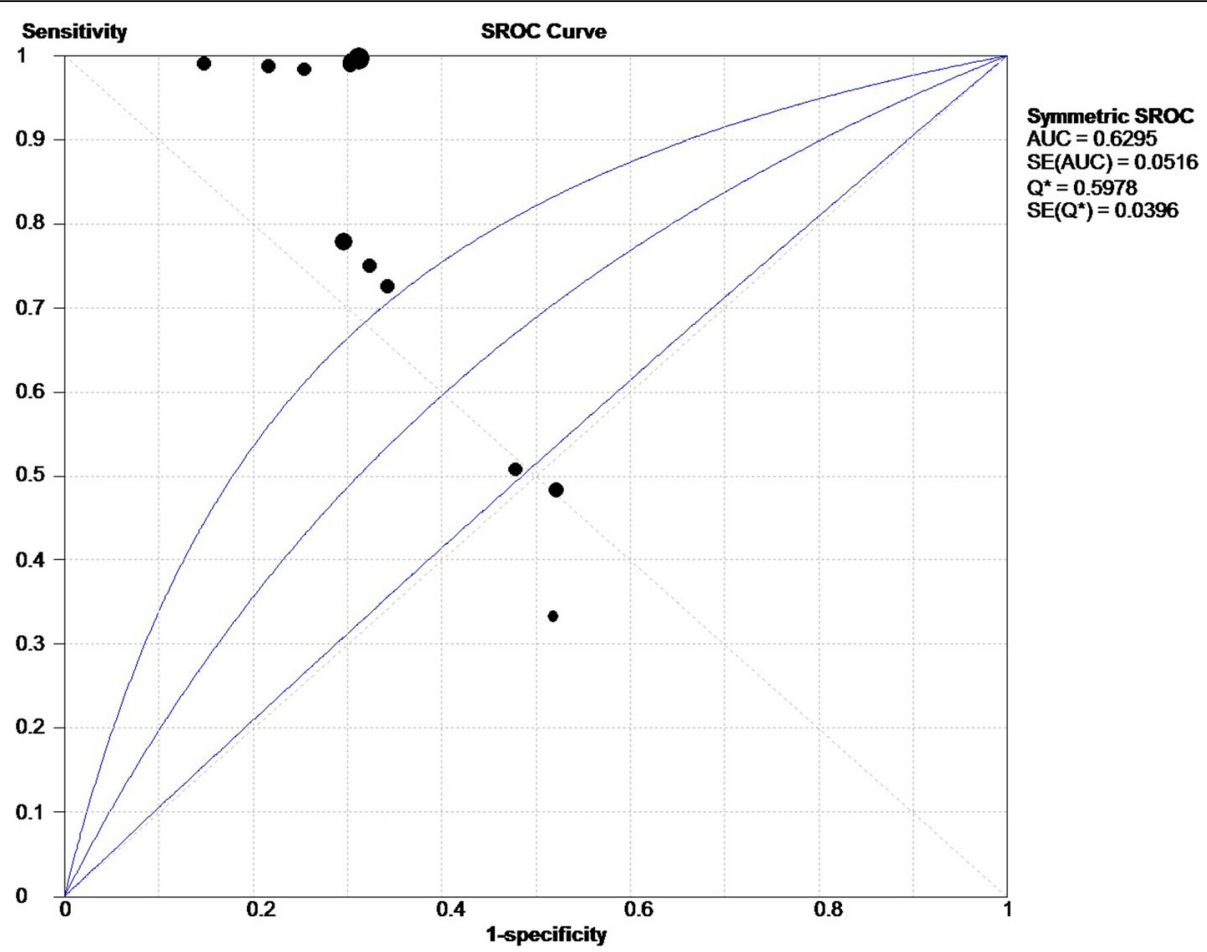

Fig. 3 Summary receiver operating characteristic (SROC) curve for VM in the diagnosis of MM cancer

and $70 \%$ specificity. The subgroup analysis was performed based on sample size ( $\leq 100$ vs. $>100$; Fig. $5 b)$. The proportion of the population with a large sample size (3 studies with more than $100 \mathrm{MM}$ cases) was 0.41 (95\% CI: $0.28-0.56 ; p=0.12$ ); while that of a sample size with less than $100 \mathrm{MM}$ patients (9 studies) was 0.31 (95\% CI: $0.23-0.41 ; p<0.001)$. Meanwhile, the highest specificity, NLR, and AUC in sample sizes less than 100 suggested that VM is more accurate in diagnosis of smaller sample sizes. Interestingly, our results show that overexpression of the VM was a high-risk prognosis factor in Asian populations (7 studies with 503 cases; $P=$ 0.32; 95\% CI: $0.23-0.42$; $p<0.001$; Fig. 5 c). As seen in
Table 3 and Fig. 5c, the pooled sensitivity and specificity were higher in the Asian patients compared to the Caucasian patients ( $85 \%$ vs. 69 and $78 \%$ vs. $68 \%$, respectively). On the other hand, we could not find any significant correlation between the $\mathrm{VM}+$ melanoma samples and gender, age, Clark level, or location of sampling (Data not shown).

\section{Publication bias and sensitivity analysis}

The publication bias and sensitivity were analyzed using Funnel plots and empirically utilizing regression tests according to Begg's rank test. The analysis was conducted by excluding a single study at a time. A symmetric inverted funnel shape in this study implies a 'well-behaved' a

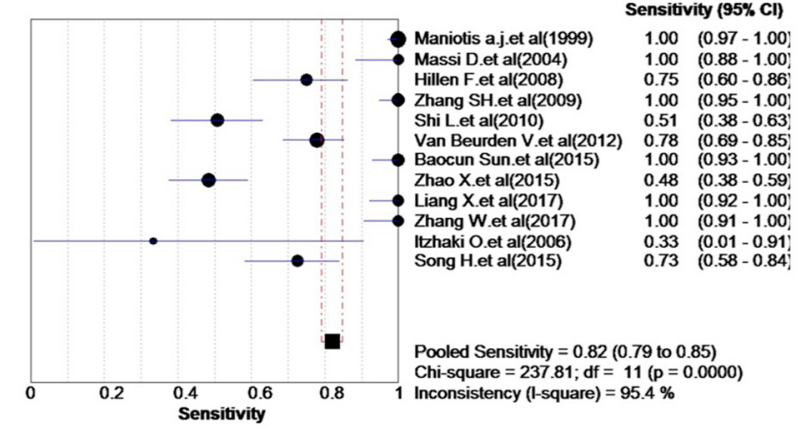

b

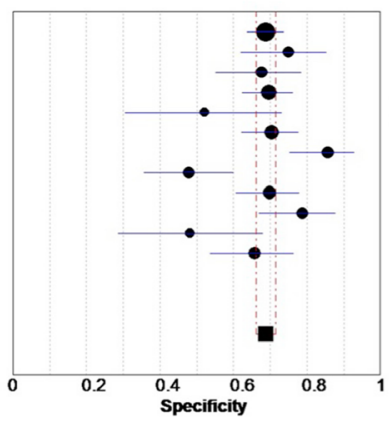

Specificity (95\% Cl)

$0.69 \quad(0.64-0.74)$ $0.75 \quad(0.62-0.85]$ $0.68 \quad(0.55-0.78$ $0.70 \quad(0.62-0.76$ $0.52 \quad(0.31-0.73$ $0.70 \quad(0.62-0.78$ $0.86 \quad(0.75-0.93$ $0.48 \quad(0.36-0.60)$ $0.70 \quad(0.61-0.78$ $0.79 \quad(0.67-0.88$ $0.48 \quad(0.29-0.68$ $0.66 \quad(0.54-0.76)$

Pooled Specificity $=0.69(0.66$ to 0.71$)$ Chi-square $=36.54 ; \mathrm{df}=11(p=0.0001)$ Inconsistency (1-square) $=69.9 \%$

Fig. 4 Forest plot of pooled sensitivity (a) and specificity (b) for VM in the diagnosis of MM cancer 


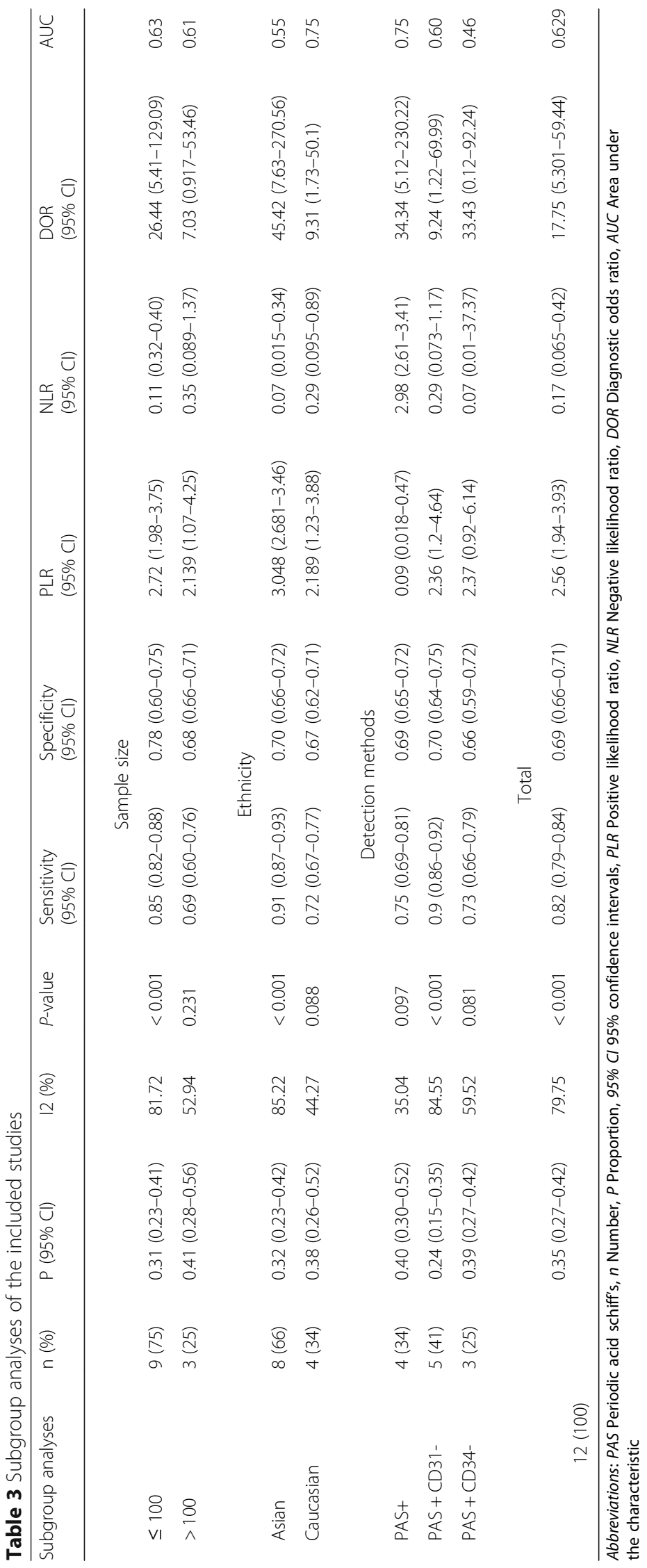



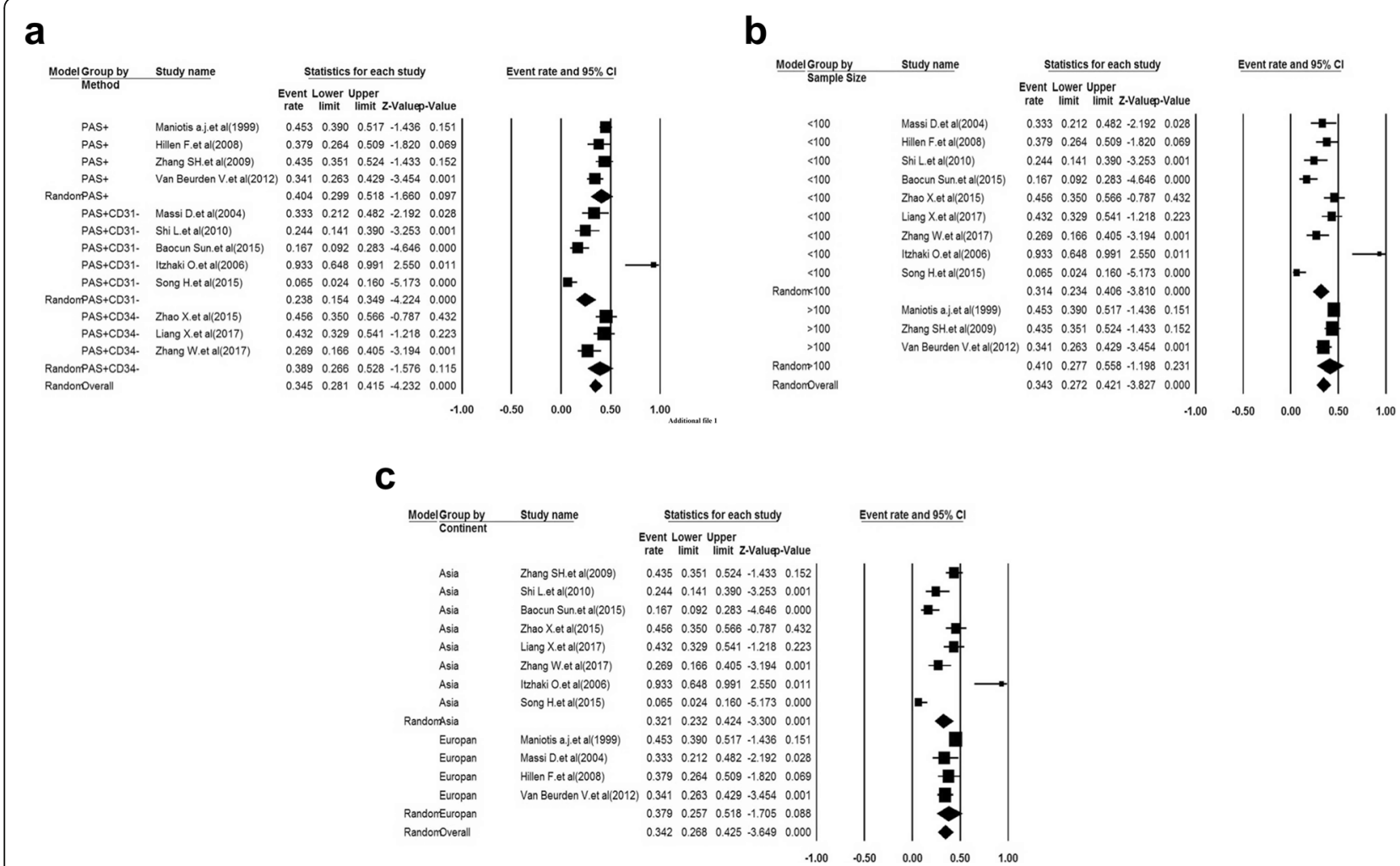

Fig. 5 Funnel plot of the sub-analysis parameters. Forest plots showed that MM cancer was associated with detection methods of VM (a), sample size (b), and race (c). Cls, confidence intervals. Weights are from random effects analysis

data set in which publication bias is improbable. Following exclusion of ten studies, there was no obvious statistical evidence for publication bias in our meta-analysis $(t=$ 1.41; $p=0.19$ ) (Fig. 6). Hence, the results of the current meta-analysis are credible and stable, with no noticeable publication bias influencing the overall results.

\section{Discussion}

To the best of our knowledge, this is the first metaanalysis study to identify the prognostic value of VM status in advanced melanoma patients. Our results indicate that $38 \%$ of MM patients with $\mathrm{VM}+$ have a poor prognosis $(P=0.35,95 \%$ CI: $0.27-0.42, p<0.001)$. Moreover, a significant association was identified in the pathologic features of the VM+ melanoma samples by race, sample size, and VM detection methods, which adversely influenced cancer survival. In the current study, the AUC of SROC was 0.63 , indicating the high accuracy of VM status as a biomarker for MM. In addition, our pooled results provided convincing evidence for a significant positive relationship between VM and small sample size.

Accumulating evidence indicates that VM is a new model of tumor microcirculation in highly aggressive malignant tumor cells $[16,17]$. Recently, in vivo and in vitro studies have shown that twist-related protein 1 (Twist1), neurogenic locus notch homolog protein 4
(Notch4), hypoxia inducible factor (HIF)-1a, EPH receptor A2 (EphA2), matrix metalloproteinase (MMP)-1, 2, $-9,-14$, and vascular endothelial (VE)-cadherin are potential therapeutic targets and prognostic indicators in $\mathrm{VM}+$ tumor samples [22, 56]. Moreover, these studies suggested that $\mathrm{VM}+$ tumor samples are resistant to common antiangiogenic drugs, such as apatinib, bevacizumab, and sunitinib $[23,34,57]$. The high ratio of neovascularization in $\mathrm{VM}+$ tumors promotes angiogenesis, metastasis, and tumor growth along with extensive hypoxia and necrosis. It also induces recruitment of various pro-angiogenic factors, such as bone marrow-derived $\mathrm{CD} 45^{+}$myeloid cells, pericyte progenitor cells, and mature $\mathrm{F} 4 / 80^{+}$tumor-associated macrophages $[58,59]$. Varying locations and heterogenic morphology of MM tumors display a close relationship with VM formation, which represents a noteworthy challenge for dermatologists $[16,60]$.

Our results clearly show that VM has a negative effect on the overall survival of MM patients with a risk ratio of 0.35 (95\% CI: $0.27-0.42, p<0.001$ ). Furthermore, our findings from sub-analyses underlined the status of VM formation in MM patients. Our results reveal a strong association between $\mathrm{VM}+$ and sample size, between $\mathrm{VM}+$ and race, as well as between $\mathrm{VM}+$ and detection method of VM $(p<0.001)$. Our findings suggest that 


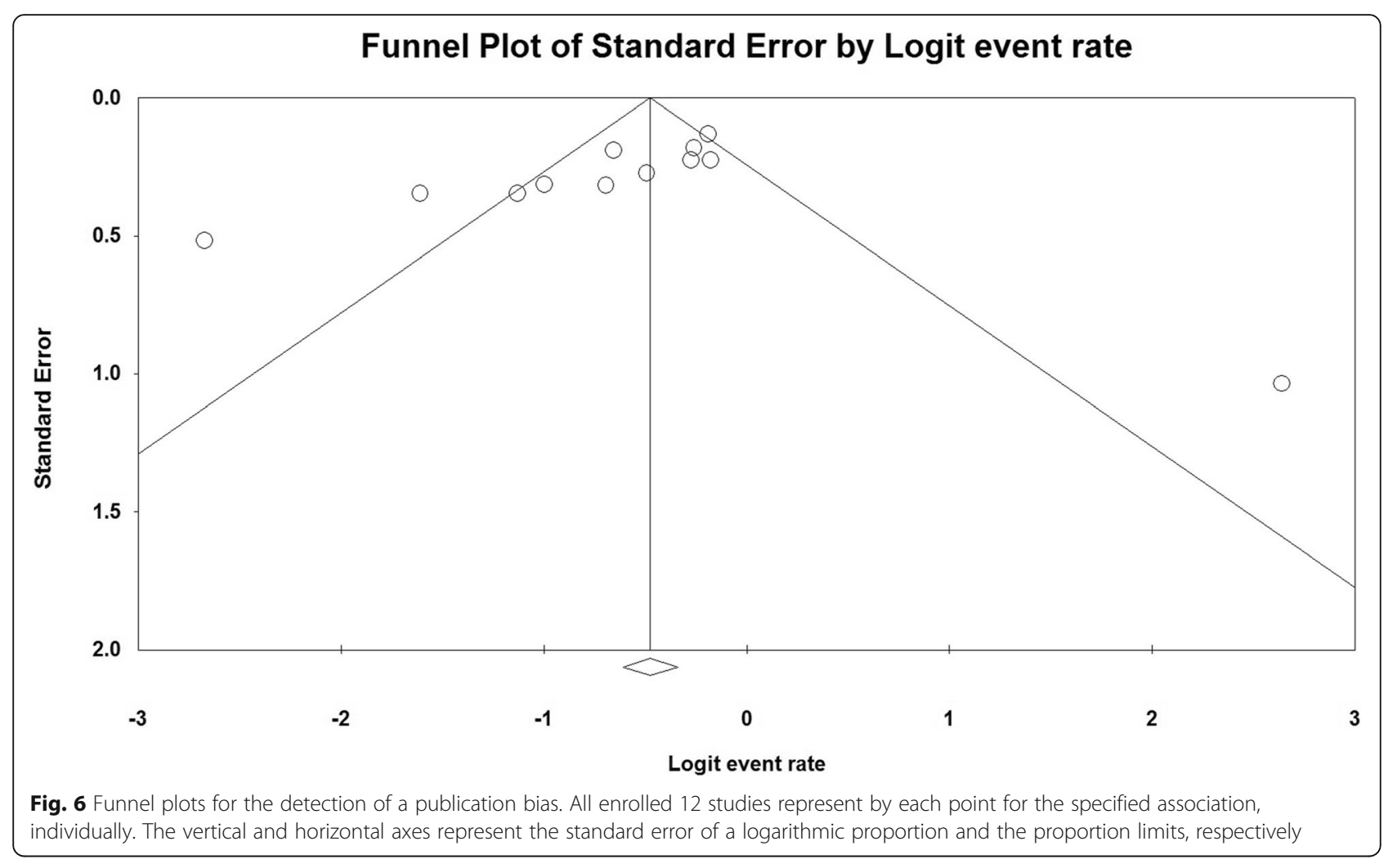

VM status can be a significantly accurate prognostic biomarker when diagnosed by CD31-/PAS+ staining, with a relatively accurate diagnostic value for VM detection (75\% sensitivity and $70 \%$ specificity). Also, the results of subgroup analyses implied a better diagnosis of VM in small sample sizes compared to that in samples containing greater than 100 cases (P: $0.31,95 \%$ CI: $0.23-0.41$; $p<0.001$ ), with a pooled sensitivity of $85 \%$ and specificity of $78 \%$. Interestingly, our results propose VM status as a more promising, accurate biomarker and target for MM diagnosis and therapeutics in Asian patients than in Caucasian patients, with a pooled sensitivity of $91 \%$ and specificity of $70.5 \%$ vs. Lifestyle factors such as UV radiation exposure and nutrition are synergistically contributing to the prevalence of MM [61, 62]. Compared to Caucasians, Asian MM patients are diagnosed at older ages; hence, in Asia we face a large population of old MM patients $[4,12]$. But considering that our study was limited to a small sample size of cases in the Caucasian group (475 cases), more large-sized studies among the Caucasian MM population should be performed to obtain a comprehensive result [61]. It is known that VM+ tumor sample profiling is more accurate in the Asian population than in the Caucasian population [62]. The meta-analysis showed that the CD31-/PAS+ staining is a more accurate detection method for $\mathrm{VM}+$ tumor samples than CD34-/PAS+ and PAS+ staining. Meanwhile, this meta-analysis suggests that postoperative detection with CD34- and/or CD31- of VM+ tumor samples in MM would be useful in finding critical therapy targets as well as for making better follow-up plans. Thus, we estimated OS in the meta-analysis, taking into account that the great majority of the studies do not report this information [62].

Several published meta-analyses have attempted to evaluate the dissimilarity of tumor VM relevant to the prognosis of cancers [27, 28, 30, 42, 63]. For example, $\mathrm{Cao}$ et al. suggested that $\mathrm{VM}+$ cancer patients have a poor 5-year overall survival rate compared to VM- cancer patients, particularly in metastatic diseases of sarcomas and lung, colon, liver, and melanoma cancers [19]. In contrast, Shen et al. addressed the tumor VM formation as an unfavorable prognostic indicator in breast cancer patients $(P=0.23$, 95\% CI: $0.08-0.38, p=0.003)$ [64]. In line with our results, Yang et al. showed that tumor VM is significantly associated with cancer differentiation, lymph node metastasis and distant metastasis, $(P=2.16 ; 95 \%$ CI: $1.98-2.38 ; p<0.001)$ [65]. With such foreground and assumptions, this current study allows us to reach a better understanding of the clinical role of $\mathrm{VM}$ formation in MM patients using statistical approaches. Conversely, the correlation between VM and survival of cancer patients remain controversial or inconclusive.

We would like to point out that there are some limitations in the current work: First, we only included papers 
published in English, while papers published in other languages, notably Chinese and Russian, were excluded, which certainly could cause selection bias. Also, we did not consider the sensitivity analysis when reflecting on the significant difference among individual articles. Importantly, in most selected studies, the common detection methods were IHC techniques. The different primary antibodies using a wide range of antibody dilutions might also affect the IHC sensitivity. Furthermore, the small sample size, short follow-up times, and lack of homogeneous distribution of the population (no studies dealing with the African continent) might also affect the precision of the estimates. However, the publication bias results showed that these limitations were not important enough to influence the analysis of late-stage and fatal complications. Future clinical studies with larger sample sizes, standardized protocols, and more homogeneous populations would be required to fully understand the prognostics potential of VM status of tumors in melanoma patients.

\section{Conclusions}

The results of the present meta-analysis suggest for the first time that VM+ status of tumors is associated with a poor OS of MM patients. We also showed that VM+ status is an accurate prognostic biomarker in small sample size groups of Asian patients. Therefore, VM status could be a promising prognostic biomarker directing surgical intervention and effective adjuvant therapy of MM patients.

\section{Supplementary information}

Supplementary information accompanies this paper at https://doi.org/10 1186/s12885-019-6350-5.

Additional file 1: Table S1. The detailed search strategy. Table S2. The excluded full-text articles. Table S3. Quality assessment of the included studies according to the Newcastle-Ottawa Scale (NOS).

Additional file 2: Figure S1. Risk of bias graph. The overall risk of bias was regarded as low in all qualified studies, in term of the QUADAS-2 assessment.

\begin{abstract}
Abbreviations
AUC: Under the curve; CAM: Comprehensive meta-analysis; Cl: Confidence interval; DOR: Diagnostic odds ratio; ECM: Extracellular matrix; EKR: Extracellular signal-regulated kinas; EphA2: EPH receptor A2; HIF1a: Hypoxia inducible factor 1a; MM: Malignant melanoma; MMP: Matrix metalloproteinase; NLR: Negative likelihood ratio; NOS: Newcastle-Ottawa scale; Notch4: Neurogenic locus notch homolog protein 4; P: Proportion; PAS: Periodic acid schiff's; PLR: Positive likelihood ratio; PRISMA: Preferred reporting items for systematic reviews and meta-analysis; QUADAS-2: Quality assessment of diagnostic accuracy studies 2; SROC: Summary receiver operating characteristic; Twist1: Twist-related protein 1; UV: Ultraviolet; VE: Vascular endothelial; VM: Vasculogenic mimicry
\end{abstract}

\section{Acknowledgements}

We thankfully acknowledge all authors for their continuous collaborations with us in sharing information. We also gratefully acknowledge all authors and coworkers for their contributions.

\section{Authors' contributions}

$\mathrm{ZZ}, \mathrm{SI}, \mathrm{MDS}$ participated in the design of the study. $\mathrm{HH}$ performed the statistical analysis. ZL, SI, and YF carried out the data extraction. SI and QW conceived of the study, and participated in its design and coordination and helped to draft the manuscript. All Authors read and approved the final manuscript.

\section{Funding}

This work was supported in part by Southwest Medical University Grant and the Research Foundation of the Science and Technology Department of Sichuan Province (No, 18080).

Availability of data and materials

Not applicable.

\section{Ethics approval and consent to participate}

This study was approved by an independent ethics committee/institutional review board at Department of Oncology, Affiliated Hospital of Southwest Medical University, Luzhou, Sichuan, China.

\section{Consent for publication}

Not applicable.

\section{Competing interests}

The authors declare that they have no competing interests.

\section{Author details}

'Department of Oncology, The Affiliated Hospital of Southwest Medical University, Luzhou City, Sichuan Province 646000, People's Republic of China. ${ }^{2}$ Department of Biology, Faculty of Science, University of Zanjan, Zanjan, Iran. ${ }^{3}$ Research Center for Evidence Based Medicine (RCEBM), Tabriz University of Medical Sciences, Tabriz, Iran.

Received: 4 July 2019 Accepted: 8 November 2019

Published online: 21 November 2019

References

1. Tas F. Metastatic behavior in melanoma: timing, pattern, survival, and influencing factors. J Oncol. 2012;2012:647684.

2. Jemal A, Saraiya M, Patel P, Cherala SS, Barnholtz-Sloan J, Kim J, Wiggins CL, Wingo PA. Recent trends in cutaneous melanoma incidence and death rates in the United States, 1992-2006. J Am Acad Dermatol. 2011;65(5 Suppl 1):S17-25.e11-13.

3. Teh YL, Goh WL, Tan SH, Yong G, Sairi ANH, Soo KC, Ong J, Chia C, Tan G, Soeharno Het al: Treatment and outcomes of melanoma in Asia: Results from the National Cancer Centre Singapore. Asia-Pacific journal of clinical oncology. 2017:4(2):95-102

4. Chang JW, Yeh KY, Wang CH, Yang TS, Chiang HF, Wei FC, Kuo TT, Yang CH. Malignant melanoma in Taiwan: a prognostic study of 181 cases. Melanoma Res. 2004;14(6):537-41.

5. Sng J, Koh D, Siong WC, Choo TB. Skin cancer trends among Asians living in Singapore from 1968 to 2006. J Am Acad Dermatol. 2009;61(3):426-32.

6. Koh D, Wang H, Lee J, Chia KS, Lee HP, Goh CL. Basal cell carcinoma, squamous cell carcinoma and melanoma of the skin: analysis of the Singapore Cancer Registry data 1968-97. Br J Dermatol. 2003;148(6):1161-6.

7. Shoo BA, Kashani-Sabet M. Melanoma arising in African-, Asian-, Latino- and native-American populations. Semin Cutan Med Surg. 2009;28(2):96-102.

8. Shoo BA, Sagebiel RW, Kashani-Sabet M. Discordance in the histopathologic diagnosis of melanoma at a melanoma referral center. J Am Acad Dermatol. 2010;62(5):751-6.

9. Kim HK, Lee S, Kim K, Heo MH, Lee H, Cho J, Kim NKD, Park W, Lee SJ, Kim $\mathrm{JH}$, et al. Efficacy of BRAF inhibitors in Asian metastatic melanoma patients: potential implications of genomic sequencing in BRAF-mutated melanoma. Transl Oncol. 2016;9(6):557-64.

10. LV J, Dai B, Kong Y, Shen X, Kong J. Acral melanoma in Chinese: a clinicopathological and prognostic study of 142 cases. Sci Rep. 2016;6: 31432.

11. Tsoutsos D, Papadopoulos S, Kehagias G, Mara A, Kyriopoulou E, Karonidis A, Spiliopoulos K, Mpalitsaris E, Karapiperis G, Panayotou P, et al. Epidemiological trends in the diagnosis of melanoma in a southern 
European population: analysis of a large database from a tertiary referral center. Melanoma Res. 2018;28(4):348-58.

12. Brunssen A, Jansen L, Eisemann N, Waldmann A, Weberpals J, Kraywinkel K, Eberle A, Holleczek B, Zeissig SR, Brenner H, et al. Longterm relative survival from melanoma in Germany 1997-2013. Melanoma Res. 2018;17(1):1-10

13. Yamazaki N, Kiyohara Y, Uhara H, Uehara J, Fujimoto M, Takenouchi T, Otsuka M, Uchi H, Ihn H, Minami H. Efficacy and safety of nivolumab in Japanese patients with previously untreated advanced melanoma: a phase II study. Cancer Sci. 2017;108(6):1223-30.

14. Mun JH, Ohn J, Kim WI, Park SM, Kim MB. Dermoscopy of melanomas on the trunk and extremities in Asians. PLoS One. 2016;11(7):e0158374.

15. Tringali C, Silvestri I, Testa F, Baldassari P, Anastasia L, Mortarini R, Anichini A, Lopez-Requena A, Tettamanti G, Venerando B. Molecular subtyping of metastatic melanoma based on cell ganglioside metabolism profiles. BMC Cancer. 2014;14:560.

16. Zhang J, Qiao L, Liang N, Xie J, Luo H, Deng G, Zhang J. Vasculogenic mimicry and tumor metastasis. J BUON. 2016;21(3):533-41.

17. Delgado-Bellido D, Serrano-Saenz S, Fernandez-Cortes M, Oliver FJ. Vasculogenic mimicry signaling revisited: focus on non-vascular VE-cadherin. Mol Cancer. 2017;16(1):65.

18. Hendrix MJ, Seftor EA, Seftor RE, Chao JT, Chien DS, Chu YW. Tumor cell vascular mimicry: novel targeting opportunity in melanoma. Pharmacol Ther. 2016;159:83-92

19. Cao Z, Bao M, Miele L, Sarkar FH, Wang Z, Zhou Q. Tumour vasculogenic mimicry is associated with poor prognosis of human cancer patients: a systemic review and meta-analysis. Eur J Cancer. 2013;49(18):3914-23.

20. Wu S, Yu L, Wang D, Zhou L, Cheng Z, Chai D, Ma L, Tao Y. Aberrant expression of CD133 in non-small cell lung cancer and its relationship to vasculogenic mimicry. BMC Cancer. 2012;12:535.

21. Abazis-Stamboulieh D, Oikonomou P, Papadoulis N, Panayiotidis P, Vrakidou E, Tsezou A. Association of interleukin-1A, interleukin-1B and interleukin-1 receptor antagonist gene polymorphisms with multiple myeloma. Leuk Lymphoma. 2007;48(11):2196-203.

22. Caramel J, Papadogeorgakis E, Hill L, Browne GJ, Richard G, Wierinckx A, Saldanha G, Osborne J, Hutchinson P, Tse G, et al. A switch in the expression of embryonic EMT-inducers drives the development of malignant melanoma. Cancer Cell. 2013;24(4):466-80.

23. Vartanian A, Baryshnikov AY. Crosstalk between apoptosis and antioxidants in melanoma vasculogenic mimicry. Adv Exp Med Biol. 2007:601:145-53.

24. Paulis YW, Soetekouw PM, Verheul HM, Tjan-Heijnen VC, Griffioen AW. Signalling pathways in vasculogenic mimicry. Biochim Biophys Acta. 2010; 1806(1):18-28.

25. Cao Z, Yu D, Fu S, Zhang G, Pan Y, Bao M, Tu J, Shang B, Guo P, Yang P, et al. Lycorine hydrochloride selectively inhibits human ovarian cancer cell proliferation and tumor neovascularization with very low toxicity. Toxicol Lett. 2013;218(2):174-85.

26. Xu XD, Yang L, Zheng LY, Pan YY, Cao ZF, Zhang ZQ, Zhou QS, Yang B, Cao C. Erratum to: Suberoylanilide hydroxamic acid, an inhibitor of histone deacetylase, suppresses vasculogenic mimicry and proliferation of highly aggressive pancreatic cancer PaTu8988 cells. BMC Cancer. 2016;16(1):374.

27. Shirakawa K, Kobayashi H, Heike Y, Kawamoto S, Brechbiel MW, Kasumi F, Iwanaga T, Konishi F, Terada M, Wakasugi H. Hemodynamics in vasculogenic mimicry and angiogenesis of inflammatory breast cancer xenograft. Cancer Res. 2002;62(2):560-6.

28. Baeten $\mathrm{Cl}$, Hillen F, Pauwels P, de Bruine AP, Baeten CG. Prognostic role of vasculogenic mimicry in colorectal cancer. Dis Colon Rectum. 2009; 52(12):2028-35.

29. Ding J, Jia X, Zuo B, He J, Yang J, He Y. A novel monoclonal antibody targeting a novel epitope of VEcadherin inhibits vasculogenic mimicry of lung cancer cells. Oncol Rep. 2018;39(6):2837-44.

30. Liu WB, Xu GL, Jia WD, Li JS, Ma JL, Chen K, Wang ZH, Ge YS, Ren WH, Yu $\mathrm{JH}$, et al. Prognostic significance and mechanisms of patterned matrix vasculogenic mimicry in hepatocellular carcinoma. Med Oncol. 2011; 28(Suppl 1):S228-38.

31. Tang HS, Feng YJ, Yao LQ. Angiogenesis, vasculogenesis, and vasculogenic mimicry in ovarian cancer. Int J Gynecol Cancer. 2009;19(4):605-10.

32. Guo Q, Yuan Y, Jin Z, Xu T, Gao Y, Wei H, Li C, Hou W, Hua B. Association between tumor vasculogenic mimicry and the poor prognosis of gastric cancer in China: an updated systematic review and meta-analysis. Biomed Res Int. 2016;2016:2408645.
33. Fujimoto A, Onodera H, Mori A, Nagayama S, Yonenaga Y, Tachibana T. Tumour plasticity and extravascular circulation in ECV304 human bladder carcinoma cells. Anticancer Res. 2006;26(1A):59-69.

34. Qiao L, Liang N, Zhang J, Xie J, Liu F, Xu D, Yu X, Tian Y. Advanced research on vasculogenic mimicry in cancer. J Cell Mol Med. 2015;19(2):315-26.

35. Panic N, Leoncini E, de Belvis G, Ricciardi W, Boccia S. Evaluation of the endorsement of the preferred reporting items for systematic reviews and meta-analysis (PRISMA) statement on the quality of published systematic review and meta-analyses. PLoS One. 2013;8(12):e83138.

36. Richardson WS, Wilson MC, Nishikawa J, Hayward RS. The well-built clinical question: a key to evidence-based decisions. ACP J Club. 1995;123(3):A12-3.

37. Stang A. Critical evaluation of the Newcastle-Ottawa scale for the assessment of the quality of nonrandomized studies in meta-analyses. Eur J Epidemiol. 2010;25(9):603-5.

38. Whiting PF, Rutjes AW, Westwood ME, Mallett S, Deeks JJ, Reitsma JB, Leeflang MM, Sterne JA, Bossuyt PM, Group Q. QUADAS-2: a revised tool for the quality assessment of diagnostic accuracy studies. Ann Intern Med. 2011;155(8):529-36.

39. Imani S, Zhang $X$, Hosseinifard H, Fu S, Fu J. The diagnostic role of microRNA-34a in breast cancer: a systematic review and meta-analysis. Oncotarget. 2017:8(14):23177-87.

40. Imani S, Hosseinifard H, Cheng J, Wei C, Fu J. Prognostic value of EMTinducing transcription factors (EMT-TFs) in metastatic breast cancer: a systematic review and meta-analysis. Sci Rep. 2016;6:28587.

41. Song H, Jing G, Wang L, Guo W, Ren G. Periodic acid-Schiff-positive loops and networks as a prognostic factor in oral mucosal melanoma. Melanoma Res. 2016;26(2):145-52.

42. Zhang W, Zhou P, Meng A, Zhang R, Zhou Y: Down-regulating Myoferlin inhibits the vasculogenic mimicry of melanoma via decreasing MMP-2 and inducing mesenchymal-to-epithelial transition. Journal of cellular and molecular medicine. 2018;22(3):1743-54.

43. Munafo MR, Clark TG, Flint J. Assessing publication bias in genetic association studies: evidence from a recent meta-analysis. Psychiatry Res. 2004;129(1):39-44

44. Egger M, Davey Smith G, Schneider M, Minder C. Bias in meta-analysis detected by a simple, graphical test. BMJ. 1997;315(7109):629-34.

45. Massi D, Franchi A, Paglierani M, Ketabchi S, Borgognoni L, Reali UM, Santucci M. Vasculogenic mimicry has no prognostic significance in PT3 and pT4 cutaneous melanoma. Hum Pathol. 2004;35(4):496-502.

46. Sun B, Zhang S, Zhao X, Zhang W, Hao X. Vasculogenic mimicry is associated with poor survival in patients with mesothelial sarcomas and alveolar rhabdomyosarcomas. Int J Oncol. 2004;25(6):1609-14.

47. Maniotis AJ, Folberg R, Hess A, Seftor EA, Gardner LM, Pe'er J, Trent JM, Meltzer PS, Hendrix MJ. Vascular channel formation by human melanoma cells in vivo and in vitro: vasculogenic mimicry. Am J Pathol. 1999;155(3):739-52.

48. Hillen F, Baeten Cl, van de Winkel A, Creytens D, van der Schaft DW, Winnepenninckx V, Griffioen AW. Leukocyte infiltration and tumor cell plasticity are parameters of aggressiveness in primary cutaneous melanoma. Cancer Immunol Immunother. 2008;57(1):97-106.

49. Zhang S, Li M, Zhang D, Xu S, Wang X, Liu Z, Zhao X, Sun B. Hypoxia influences linearly patterned programmed cell necrosis and tumor blood supply patterns formation in melanoma. Lab Invest. 2009;89(5): $575-86$.

50. Shi L, Lei D, Ma C, Xu F, Li Y, Wang Y, Cong N, Liu D, Pan XL. Clinicopathological implications of tumour-associated macrophages and vascularization in sinonasal melanoma. J Int Med Res. 2010;38(4):1276-86.

51. van Beurden A, Schmitz RF, van Dijk CM, Baeten Cl. Periodic acid Schiff loops and blood lakes associated with metastasis in cutaneous melanoma. Melanoma Res. 2012;22(6):424-9.

52. Baocun S, Shiwu Z, Xiulan Z, Danfang Z, Xishan H. Pilot study of molecular mechanism on vasculogenic mimicry in bi-directional differentiated malignant tumors. Chin-Ger J Clin Oncol. 2015;4(1):50-2.

53. Zhao X, Sun B, Li Y, Liu Y, Zhang D, Wang X, Gu Q, Zhao J, Dong X, Liu Z, et al. Dual effects of collagenase-3 on melanoma: metastasis promotion and disruption of vasculogenic mimicry. Oncotarget. 2015;6(11):8890-9.

54. Liang $X$, Sun $R$, Zhao $X$, Zhang $Y$, Gu Q, Dong $X$, Zhang D, Sun J, Sun B. Rictor regulates the vasculogenic mimicry of melanoma via the AKT-MMP2/9 pathway. J Cell Mol Med. 2017;21(12):3579-91.

55. Itzhaki O, Greenberg E, Shalmon B, Kubi A, Treves AJ, Shapira-Frommer R, Avivi C, Ortenberg R, Ben-Ami E, Schachter J, et al. Nicotinamide inhibits 
vasculogenic mimicry, an alternative vascularization pathway observed in highly aggressive melanoma. PLoS One. 2013;8(2):e57160.

56. Liu Q, Qiao L, Liang N, Xie J, Zhang J, Deng G, Luo H, Zhang J. The relationship between vasculogenic mimicry and epithelial-mesenchymal transitions. J Cell Mol Med. 2016;20(9):1761-9.

57. Francis JH, Kim J, Lin A, Folberg R, lyer S, Abramson DH. Growth of uveal melanoma following intravitreal bevacizumab. Ocul Oncol Pathol. 2017;3(2):117-21.

58. Schnegg $\mathrm{Cl}$, Yang MH, Ghosh SK, Hsu MY. Induction of vasculogenic mimicry overrides VEGF-A silencing and enriches stem-like cancer cells in melanoma. Cancer Res. 2015;75(8):1682-90.

59. Liu J, Qu X, Shao L, Hu Y, Yu X, Lan P, Guo Q, Han Q, Zhang J, Zhang C. Pim-3 enhances melanoma cell migration and invasion by promoting STAT3 phosphorylation. Cancer Biol Ther. 2018;19(3):160-8.

60. Pulford E, Hocking A, Griggs K, McEvoy J, Bonder C, Henderson DW, Klebe S. Vasculogenic mimicry in malignant mesothelioma: an experimental and immunohistochemical analysis. Pathology. 2016;48(7):650-9.

61. Chen L, Jin S. Trends in mortality rates of cutaneous melanoma in east Asian populations. PeerJ. 2016;4:e2809.

62. Matthews NH, Li WQ, Qureshi AA, Weinstock MA, Cho E. Epidemiology of melanoma. In: Ward WH, Farma JM, editors. Cutaneous melanoma: etiology and therapy. edn. Brisbane: Codon Publications; 2017.

63. Qin L, Ren Y, Chen AM, Guo FJ, Xu F, Gong C, Cheng P, Du Y, Liao H. Peroxisome proliferator-activated receptor gamma ligands inhibit VEGFmediated vasculogenic mimicry of prostate cancer through the AKT signaling pathway. Mol Med Rep. 2014;10(1):276-82.

64. Shen Y, Quan J, Wang M, Li S, Yang J, Lv M, Chen Z, Zhang L, Zhao X, Yang J. Tumor vasculogenic mimicry formation as an unfavorable prognostic indicator in patients with breast cancer. Oncotarget. 2017;8(34):56408-16.

65. Yang JP, Liao YD, Mai DM, Xie P, Qiang YY, Zheng LS, Wang MY, Mei Y, Meng DF, Xu L, et al. Tumor vasculogenic mimicry predicts poor prognosis in cancer patients: a meta-analysis. Angiogenesis. 2016;19(2):191-200.

\section{Publisher's Note}

Springer Nature remains neutral with regard to jurisdictional claims in published maps and institutional affiliations.

Ready to submit your research? Choose BMC and benefit from:

- fast, convenient online submission

- thorough peer review by experienced researchers in your field

- rapid publication on acceptance

- support for research data, including large and complex data types

- gold Open Access which fosters wider collaboration and increased citations

- maximum visibility for your research: over $100 \mathrm{M}$ website views per year

At $\mathrm{BMC}$, research is always in progress.

Learn more biomedcentral.com/submissions 